\title{
Si les anticorps anti-ADN n'étaient pas dirigés primitivement contre I'ADN...
}

Les nouvelles de ce numéro ont été préparées par : J. P. Grunfeld A. Kahn

A. Berthelot J. C. Dreyfus S. Erlinger
Cette rubrique est destinée à offrir des informations possédant un intérêt général. Les nouvelles feront état de publications récentes, datant des dernières semaines mais parfois aussi de quelques mois, lorsque leur décantation permet mieux d'en établir l'importance. De courtes mises au point sur un sujet d'actualité pourront également être présentées. En outre, nous sommes conscients que le développement des techniques de la biologie moléculaire a fait naître un langage nouveau, jargon professionnel qui risquerait d'en détourner les esprits même les plus intéressés. Cette crainte nous a incités à proposer une sorte de "lexique" " qui paraîtra dans les premiers numéros de la revue, et dont on trouvera le premier exemple en page suivante. a responsabilité des anticorps anti-ADN natifs double brin dans les lésions observées au cours du lupus érythémateux disséminé (LED) a été suggérée par de nombreux auteurs. Ces anticorps sont en effet rendus responsables d'un grand nombre de lésions tissulaires, en particulier rénales, par l'intermédiaire du dépôt de complexes immuns ADN-anti-ADN. De plus, il représentent un bon marqueur de la maladie. Néanmoins, la présence d'ADN au sein de ces complexes immuns n'a pas pu être démontrée formellement.

Pour apprécier le caractère homogène ou hétérogène de la population d'anticorps anti-ADN présente dans le sérum de patients atteints de LED et de celui des souris $\mathrm{B} / \mathrm{W}$ qui réalisent une maladie en tous points comparable au LED humain, des anticorps anti-ADN monoclonaux ont été produits par fusion entre une rate de souris $\mathrm{B} / \mathrm{W}$ auto-immune, non immunisée et un myélome non secrétant de souris $\mathrm{BALB} / \mathrm{C}$

L'utilisation de ces anticorps monoclonaux a permis d'aborder ce qui peut être considéré comme le paradoxe fondamental de la maladie lupique : d'une part, des anticorps anti-ADN sont spontanément produits en grande quantité au cours du LED humain et murin et d'autre part, il est particulièrement difficile de produire par immunisation des anticorps contre l'ADN. Or, il a été récemment montré que des anticorps monoclonaux spécifiquement dirigés contre l'ADN double brin reconnaissent une ou des protéines, en voie de caractérisation biochimique et d'isolement, situées à la surface des différentes lignées cellulaires humaines touchées dans le LED : glomérules, lymphocytes $\mathrm{B}$ et $\mathrm{T}$, érythrocytes, plaquettes et système nerveux central. Fait important, ces anticorps reconnaissent la ou les même (s) protéine $(\mathrm{s})$ à la surface de ces différents types cellulaires. En revanche, l'intestin, le pancréas, et le foie rarement atteints dans le LED ne sont pas reconnus par ces anticorps.

Ces résultats suggèrent qu'il existe donc un motif antigénique commun entre l'ADN double brin et cette (ou ces) protéine (s) présente (s) à la surface de différents types cellulaires humains concernés dans le LED. Cette conclusion est d'autant plus inattendue qu'il était admis que les anticorps anti-ADN reconnaissaient le noyau des cellules et non pas les membranes cytoplasmiques. En fait, la question se pose désormais de savoir si la (ou les) protéines (s) en question plutôt que l'ADN ne joue (nt) pas le rôle d'immunogène dans le LED. On peut aussi se demander si les lésions tissulaires, en particulier rénales, observées avec une grande fréquence dans la maladie ne sont pas expliquées par une action directe des anticorps sur ces tissus plutôt que par le dépôt de complexes immuns ADN-anti-ADN.

Finalement, ces résultats suggèrent que l'antigène initiateur et l'antigène cible du LED pourraient constituer une structure complètement distincte de l'ADN telle qu'une protéine de surface cellulaire bien que longtemps confondu avec l'ADN car donnant lieu à des réactions croisées. J. P. G.

Jacob L, Lety MA, Louvard P, Bach JF. Binding of a monoclonal anti-1). I autoantibody to identical protein (s) present at the surface of different cell types involved in lupus pathogenesis 7 Clin Invest 1985; $75: 315-7$.
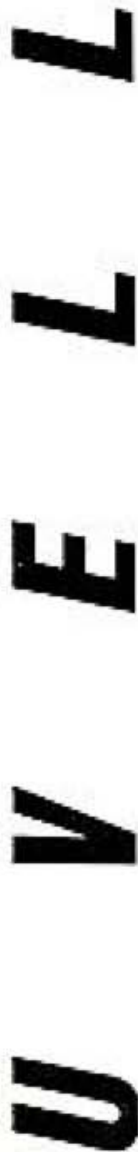